\title{
A Generalised Entropy Function
}

\author{
J. Naudts \\ Universiteit Leuven, Leuven, Belgium
}

Received December 6, 1973

\begin{abstract}
Let $\varphi$ be a faithful normal semi-finite weight on a von Neumann algebra $\mathscr{M}$. Normal states on $\mathscr{M}$ almost majorised by this weight are defined. For this class of states on $\mathscr{M}$ a theorem is proved. Using this result we define entropy of normal states on $\mathscr{M}$ and we show that this entropy function generalises the entropy both of classical and of quantum statistical mechanics.
\end{abstract}

\section{Introduction}

Let $\varphi$ and $\psi$ be two normal states on a von Neumann algebra $\mathscr{M}$. Suppose $\psi$ is faithful. In Ref. [1] Dixmier introduces the notion of the state $\varphi$ being almost majorised by the state $\psi$. He remarks that to any state $\varphi$ almost majorised by $\psi$ corresponds a closable operator affiliated with $\pi_{\psi}(\mathscr{M})^{\prime}$, where $\pi_{\psi}$ is the *-representation of $\mathscr{M}$ associated with $\psi$ by the G.N.S.-construction.

We define when a normal state $\varphi$ on $\mathscr{M}$ is almost majorised by a faithful normal semi-finite weight $\psi$ on $\mathscr{M}$.

Using some results of Perdrizet [2], we show that with any state $\varphi$ almost majorised by the weight $\psi$ can be associated in a unique way a positive self-adjoint operator affiliated with $\pi_{\psi}(\mathscr{M})^{\prime}$.

This result is used to define a generalised entropy function. The phase space of a system in classical statistical mechanics is a measure space $M, v$. The measure $v$ gives the a priori probability of the points of $M$. The macroscopic states of the system are described by positive normalised measures $\mu$ on $M$ which are absolutely continuous with respect to the measure $v$. To each such measure $\mu$ corresponds a positive integrable function $f$ on $M$ which satisfies $\int f d v=1$ and $d \mu=f d v$. These functions $f$ are called density functions and the entropy of the measure $\mu$ is given by the expression

$$
S(\mu)=-\int f \log f d v .
$$

Let $\mathscr{H}$ be the Hilbert space of wave functions of a quantum mechanical system. In many cases the statistical states of the system are described by the normal states on the space $\mathscr{B}(\mathscr{H})$ of all bounded linear operators on $\mathscr{H}$. To each normal state $\psi$ on $\mathscr{B}(\mathscr{H})$ corresponds a unique density 
matrix on $\mathscr{H}$, i.e.: a positive trace class operator $\varrho$ on $\mathscr{H}$ which satisfies $\operatorname{Tr} \varrho=1$ and $\psi(A)=\operatorname{Tr} \varrho A$ for all $A \in \mathscr{B}(\mathscr{H})$. The entropy $S(\psi)$ of the normal state $\psi$ on $\mathscr{B}(\mathscr{H})$ is defined by:

$$
S(\psi)=-\operatorname{Tr} \varrho \log \varrho .
$$

This entropy function is the quantum analogue of the classical entropy function defined above. However it should be pointed out how restrictive this quantum mechanical definition is. For classical systems we have free choice of the a priori probability measure with respect to which entropy is calculated. In the definition of entropy for quantum systems the trace plays the role of the a priori probability measure and no choice is made.

Let $\mathscr{M}$ be a von Neumann algebra with a faithful normal semifinite weight $\varphi$ on it. In analogy with the density functions $f$ on the phase space $M, v$ of a classical system and with the density matrices $\varrho$ on the Hilbert-space $\mathscr{H}$ of wave functions of a quantum system we call density operators the positive self-adjoint operators $T$ associated with the normal states on $\mathscr{M}$ which are almost majorised by $\varphi$.

If $\mathscr{M}$ is the $W^{*}$-algebra $\mathscr{L}^{\infty}(M, v)$ of all essentially bounded measurable functions on the measure space $M, v$, a weight $\varphi$ on $\mathscr{M}$ is defined by the relation

$$
\varphi\left(A^{*} A\right)=\int A^{*} A d v \text { for all } A \text { in } \mathscr{M} .
$$

Then we may show that there is a one-to-one correspondence between density functions on $M, v$ and density operators affiliated with $\pi_{\varphi}(\mathscr{M})^{\prime}$.

If $\mathscr{M}$ equals the space $\mathscr{B}(\mathscr{H})$ of all bounded linear operators on a Hilbert space $\mathscr{H}$ one weight $\varphi$ on $\mathscr{M}$ is e.g. the trace. Then there is a one-to-one correspondence between density matrices on $\mathscr{H}$ and density operators affiliated with $\pi_{\varphi}(\mathscr{M})^{\prime}$.

In both cases $\mathscr{M}=\mathscr{L}^{\infty}(M, v)$ and $\mathscr{M}=\mathscr{B}(\mathscr{H})$ we express the entropy $S(\mu)$ of a measure $\mu$ on $M$ resp. the entropy $S(\psi)$ of a normal state $\psi$ on $\mathscr{B}(\mathscr{H})$ in terms of density operators and twice we find the same expression. This justifies the general definition of entropy we introduce at the end of Chapter 3.

We now give a short mathematical introduction (see [5]). A weight on a $C^{*}$-algebra $\mathscr{A}$ is a function $\varphi: \mathscr{A}^{+} \rightarrow[0,+\infty]$ satisfying:

$$
\begin{aligned}
\varphi(A+B) & =\varphi(A)+\varphi(B) & & \text { for all } A, B \text { in } \mathscr{A}^{+} \\
\varphi(\alpha A) & =\alpha \varphi(A) & & \text { for all } \alpha \geqq 0 \text { and for all } A
\end{aligned}
$$

in $\mathscr{A}^{+}$(with the convention $0, \infty=0$ ).

Define:

$$
\mathfrak{N}_{\varphi}=\left\{A \in \mathscr{A} \mid \varphi\left(A^{*} A\right)<+\infty\right\} .
$$

Then $\mathfrak{X}_{\varphi}$ is a left ideal in $\mathscr{A}$. 
Theorem. With any weight $\varphi$ on a $C^{*}$-algebra $\mathscr{A}$ is associated a Hilbert space $\mathscr{H}_{\varphi}$ and two mappings $\Lambda_{\varphi}$ and $\pi_{\varphi}$, where $\Lambda_{\varphi}$ is linear from $\mathfrak{N}_{\varphi}$ into $\mathscr{H}_{\varphi}$ and $\pi_{\varphi}$ is a $*$-representation of $\mathscr{A}$ into $\mathscr{B}\left(\mathscr{H}_{\varphi}\right)$ such that $\left(\pi_{\varphi}(A) \Lambda_{\varphi} B, \Lambda_{\varphi} C\right)=\varphi\left(C^{*} A B\right)$ for all $A$ in $\mathscr{A}$ and $B, C$ in $\mathfrak{N}_{\varphi}$.

A weight $\varphi$ on a von Neumann algebra $\mathscr{M}$ is normal if for any increasing net $\left(A_{\alpha}\right)_{\alpha}$ in $\mathscr{M}^{+}$with l.u.b. $A$ in $\mathscr{M}^{+}$we have $\varphi(A)=$ l.u.b. $\varphi\left(A_{\alpha}\right)$ (see [6]).

Theorem. If $\varphi$ is a faithful normal semi-finite weight on a von Neumann algebra $\mathscr{M}$ then the set $\mathscr{A}_{\varphi}=\mathfrak{N}_{\varphi} \cap \mathfrak{N}_{\varphi}^{*}$ is a full left Hilbert algebra with $\mathscr{M}$ as its left von Neumann algebra.

I'm indebted to Prof. H. Araki and Prof. A. Van Daele for simplifications in the proof of Theorem 2.3.

\section{Density Operators}

Definition 2.1. Let $\varphi$ be a normal weight on a von Neumann algebra $\mathscr{M}$. A positive selfadjoint operator $T$ affiliated with $\pi_{\varphi}(\mathscr{M})^{\prime}$ is a density operator with respect to $\varphi$ if $\Lambda_{\varphi} \mathfrak{N}_{\varphi}$ is a core of $T^{\frac{1}{2}}$ (i.e. the closure of the restriction of $T^{\frac{1}{2}}$ to $\Lambda_{\varphi} \mathfrak{N}_{\varphi}$ again is $T^{\frac{1}{2}}$ ) and if there exists a normal state $\psi$ on $\mathscr{M}$ such that $\psi\left(B^{*} A\right)=\left(T^{\frac{1}{2}} \Lambda_{\varphi} A, T^{\frac{1}{2}} \Lambda_{\varphi} B\right)$ for all $A, B$ in $\mathfrak{N}_{\varphi}$.

The following definition generalises a definition of Dixmier $([1]$, I, 4, Exercise 8):

Definition 2.2. Let $\varphi$ be a normal weight on a von Neumann algebra $\mathscr{M}$. A normal state $\psi$ on $\mathscr{M}$ is almost majorised by $\varphi$ if for any net $\left(A_{\alpha}\right)_{\alpha}$ of elements in $\mathfrak{N}_{\varphi}$ such that $\varphi\left(A_{\alpha}^{*} A_{\alpha}\right) \rightarrow 0, \psi\left(\left(A_{\alpha}-A_{\beta}\right)_{*}\left(A_{\alpha}-A_{\beta}\right)\right) \underset{\alpha, \beta \rightarrow \infty}{\longrightarrow} 0$ implies $\psi\left(A_{\alpha}^{*} A_{\alpha}\right) \rightarrow 0$.

The following theorem generalises Dixmier [1], I, 4, Exercise 8, and Perdrizet [2], p. 45, Proposition 6.4:

Theorem 2.3. Let $\varphi$ be a faithful normal semi-finite weight on a von Neumann algebra $\mathscr{M}$ and $\psi$ a normal state on $\mathscr{M}$. Then the following conditions are equivalent:

a) $\psi$ is almost majorised by $\varphi$.

b) There exists a vector ' $\xi$ in $\mathscr{H}_{\varphi}$ such that $\psi(A)=\left(\pi_{\phi}(A) \xi\right.$, $\left.\xi\right)$ for all $A \in \mathscr{M}$ and for all such $\xi$ the operator $\varrho^{\prime}(\xi): \Lambda_{\varphi} A \rightarrow \pi_{\varphi}(A) \xi$ with domain $\Lambda_{\varphi} \mathfrak{N}_{\varphi}$ is closable.

c) There exists a density operator $T$ with respect to $\varphi$ such that $\psi\left(B^{*} A\right)=\left(T^{\frac{1}{2}} \Lambda_{\varphi} A, T^{\frac{1}{2}} \Lambda_{\varphi} B\right)$ for all $A, B$ in $\mathfrak{N}_{\varphi}$.

This density operator $T$ is uniquely determined by the state $\Psi$.

Proof. a) $\Rightarrow$ b). 
The existence of a vector $\xi$ in $\mathscr{H}_{\varphi}$ such that $\psi(A)=\left(\pi_{\varphi}(A) \xi, \xi\right)$ for all $A \in \mathscr{M}$ follows from [2], p. 44, Proposition 6.2.

Let $\left(A_{\alpha}\right)_{\alpha}$ be a net of elements in $\mathfrak{N}_{\varphi}$ such that $\Lambda_{\varphi} A_{\alpha} \rightarrow 0$ and $\left(\pi_{\varphi}\left(A_{\alpha}\right) \xi\right)_{\alpha}$. is convergent. This means that $\varphi\left(A_{\alpha}^{*} A_{\alpha}\right) \rightarrow 0$ and that $\psi\left(\left(A_{\alpha}-A_{\beta}\right)^{*}\right.$ $\left.\left(A_{\alpha}-A_{\beta}\right)\right) \underset{\alpha, \beta \rightarrow \infty}{\longrightarrow} 0$. Using condition a) there follows that $\psi\left(A_{\alpha}^{*} A_{\alpha}\right) \rightarrow 0$ or that $\pi_{\varphi}\left(A_{\alpha}\right) \xi \rightarrow 0$. This shows that the operator $\varrho^{\prime}(\xi): \Lambda_{\varphi} A \rightarrow \pi_{\varphi}(A) \xi$ is closable.

b) $\Rightarrow$ c).

Let $\xi \in \mathscr{H}_{\varphi}$ be such that $\Psi(A)=\left(\pi_{\varphi}(A) \xi, \xi\right)$ for any $A \in \mathscr{M}$.

By b) the operator $\varrho^{\prime}(\xi)$ is closable.

Let $T=\varrho^{\prime}(\xi) * \overline{\varrho^{\prime}(\xi)}$. Let $\overline{\varrho^{\prime}(\xi)}=U T^{\frac{1}{2}}$ be the polar decomposition of $\overline{\varrho^{\prime}(\xi)}$. Then $U^{*} U T^{\frac{1}{2}}=T^{\frac{1}{2}}$, which implies

$$
\Psi\left(B^{*} A\right)=\left(T^{\frac{1}{2}} \Lambda_{\varphi} A, T^{\frac{1}{2}} \Lambda_{\varphi} B\right) \text { for any } A \text { and } B \text { in } \mathfrak{N}_{\varphi} .
$$

For any $A \in \mathscr{M}$ and $B \in \mathfrak{N}_{\varphi}$ one has $\varrho^{\prime}(\xi) \pi_{\varphi}(A) \Lambda_{\varphi} B=\pi_{\varphi}(A) \varrho^{\prime}(\xi) \Lambda_{\varphi} B$. Hence $\varrho^{\prime}(\xi) \pi_{\varphi}(A) \supset \pi_{\varphi}(A) \varrho^{\prime}(\xi)$. This implies the operator $\frac{\varrho^{\prime}(\xi)}{\varrho^{\prime}}$ is affiliated with $\pi_{\varphi}(\mathscr{M})^{\prime}\left([1], \mathrm{I}, \S 3\right.$, Exercise 7 a). Hence $T$ is affiliated with $\pi_{\varphi}(\mathscr{M})^{\prime}$.

Since any core of $\overline{\varrho^{\prime}(\xi)}$ is a core of $T^{\frac{1}{2}}, \Lambda_{\varphi} \mathfrak{N}_{\varphi}$ is a core of $T^{\frac{1}{2}}$. We conclude $T$ is a density operator corresponding to the state $\Psi$.

c) $\Rightarrow$ a).

Let $\left(A_{\alpha}\right)_{\alpha}$ be a net of elements in $\mathfrak{N}_{\varphi}$ for which $\varphi\left(A_{\alpha}^{*} A_{\alpha}\right) \rightarrow 0$ and $\psi\left(\left(A_{\alpha}-A_{\beta}\right)_{*}\left(A_{\alpha}-A_{\beta}\right)\right) \underset{\alpha, \beta \rightarrow \infty}{\longrightarrow} 0$. Then $\Lambda_{\varphi} A_{\alpha} \rightarrow 0$ and $\left(T^{\frac{1}{2}} \Lambda_{\varphi} A_{\alpha}\right)_{\alpha}$ converges. Because the operator $T^{\frac{1}{2}}$ is closed $T^{\frac{1}{2}} \Lambda_{\varphi} A_{\alpha}$ converges to zero. Hence $\psi\left(A_{\alpha}^{*} A_{\alpha}\right) \rightarrow 0$.

Let $S$ and $T$ be density operators corresponding to the state $\Psi$.

Then for any $A \in \mathfrak{N}_{\varphi}$ :

$$
\left\|S^{\frac{1}{2}} \Lambda_{\varphi} A\right\|^{2}=\Psi\left(A^{*} A\right)=\left\|T^{\frac{1}{2}} \Lambda_{\varphi} A\right\|^{2} .
$$

Hence there exists a partical isometry $U$ from the closed range of $S^{\frac{1}{2}}$ onto the closed range of $T^{\frac{1}{2}}$ such that for any $A \in \mathfrak{N}_{\varphi}$

$$
U S^{\frac{1}{2}} \Lambda_{\varphi} A=T^{\frac{1}{2}} \Lambda_{\varphi} A
$$

Because $\Lambda_{\varphi} \mathfrak{N}_{\varphi}$ is a core of $S^{\frac{1}{2}}$ and of $T^{\frac{1}{2}}$ one obtains $U S^{\frac{1}{2}} \supset T^{\frac{1}{2}}$ and $S^{\frac{1}{2}} C U^{*} T^{\frac{1}{2}}$ i.e. $U S^{\frac{1}{2}}=T^{\frac{1}{2}}$.

Now $S^{\frac{1}{2}}$ and $T^{\frac{1}{2}}$ both are positive selfadjoint operators. Because of the uniqueness of the polar decomposition one has $U=\mathbb{1}$ and $S^{\frac{1}{2}}=T^{\frac{1}{2}}$.

Q.E.D.

This theorem characterises the states on the von Neumann algebra $\mathscr{M}$ for which a density operator exists. It has been shown that not all normal states on $\mathscr{M}$ are almost majorised by a given faithfull normal weight $\varphi([2]$, p. 45, Remark 6.5, (1)). 
Corollary 2.4. Let $T$ be a density operator with respect to the normal weight $\varphi$. There exists a unique vector $\xi$ in $\mathscr{H}_{\varphi}$ for which $\varrho^{\prime}(\xi)$ is closable and the closure $\pi^{\prime}(\xi)$ of $\varrho^{\prime}(\xi)$ equals $T^{\frac{1}{2}}$.

Proof. Let $\Psi^{\prime}$ be a normal state on $\mathscr{M}$ satisfying

$$
\Psi\left(B^{*} A\right)=\left(T^{\frac{1}{2}} \Lambda_{\varphi} A, T^{\frac{1}{2}} \Lambda_{\varphi} B\right) \text { for all } A, B \text { in } \mathfrak{N}_{\varphi} .
$$

From the proof of foregoing theorem follows there exists a $\zeta \in \mathscr{H}_{\varphi}$ such that $\overline{\varrho^{\prime}(\zeta)}$ has a polar decomposition of the form $\overline{\varrho^{\prime}(\zeta)}=U T^{\frac{1}{2}}$. Now one has: $\varrho^{\prime}\left(U^{*} \zeta\right)=U^{*} \varrho^{\prime}(\zeta)$.

Hence: $\overline{\varrho^{\prime}(\xi)}=T^{\frac{1}{2}}$ with $\xi=U^{*} \zeta$.

Suppose $\xi_{1}$ and $\xi_{2} \in \mathscr{H}_{\varphi}$ satisfy $\pi^{\prime}\left(\xi_{1}\right)=\pi^{\prime}\left(\xi_{2}\right)$.

Then $\varrho^{\prime}\left(\xi_{1}\right)=\varrho^{\prime}\left(\xi_{2}\right)$ which implies $\xi_{1}=\xi_{2}$.

\section{Entropy}

Let $M, v$ be a localisable measure space ([3]). Then the predual of the $W^{*}$-algebra $\mathscr{L}^{\infty}(M, v)$ of all essentially bounded measurable functions on $M, v$ is the space $\mathscr{L}^{1}(M, v)$ of all integrable functions ([4], p. 45, 1.18). Hence there is a one-to-one correspondence between the positive normalised measures $\mu$ on $M$ absolutely continuous with respect to $v$ and the normal states $\psi_{\mu}$ on $\mathscr{L}^{\infty}(M, v)$. The measure $v$ defines a weight $\varphi$ on $\mathscr{L}^{\infty}(M, v)$ which is faithful, normal and semi-finite.

If $T$ is a density operator with respect to $\varphi$ and $\xi$ is the unique vector in $\mathscr{H}_{\varphi}$ for which $T=\pi^{\prime}(\xi)^{2}$ then we introduce following notation:

$\langle\log T\rangle_{T}=\lim _{\lambda \rightarrow \infty}\left(\left(\log T F_{\lambda}\right) \xi, \xi\right)$ whenever this limit converges. The $F_{\lambda}$ are determined by:

$$
T=\int_{0}^{+\infty} \lambda d E_{\lambda} \text { is the spectral decomposition of } T \text { and } F_{\lambda}=\int_{1 / \lambda}^{\lambda} d E_{\lambda} \text {. }
$$

Proposition 3.1. Let $\mu$ be a positive normalised measure on the localisable measure space $M, v$. Suppose $\mu$ is absolutely continuous with respect to $v$. Then the normal state $\psi_{\mu}$ on $\mathscr{L}^{\infty}(M, v)$ is almost majorised by the weight $\varphi$. Let $T_{\mu}$ be the unique density operator associated with $\psi_{\mu}$. Then the entropy $S(\mu)$ is defined if and only if $\left\langle\log T_{\mu}\right\rangle_{T_{\mu}}$ exists and in that case:

$$
S(\mu)=-\left\langle\log T_{\mu}\right\rangle_{T_{\mu}} .
$$

Proof. Identify $\mathscr{L}^{\infty}(M, v)$ with its representation $\pi_{\varphi}\left(\mathscr{L}^{\infty}(M, v)\right)$.

Let $f$ be the positive integrable function for which $d \mu=f d v$. Denote by $N_{\lambda}$, for $\lambda \geqq 0$, the set

$$
N_{\lambda}=\{x \in M \mid f(x) \leqq \lambda\} .
$$

Then the sets $N_{\lambda}$ are measurable and the characteristic functions $E_{\lambda}=\chi\left(N_{\lambda}\right)$ are projections in $\mathscr{L}^{\infty}(M, v)$. 
The $\left(E_{\lambda}\right)_{\lambda}$ form a spectral family, i.e. they satisfy:

a) $E_{\lambda} \leqq E_{\mu}$ if $\lambda<\mu$,

b) $E_{\lambda}=\underset{\mu \rightarrow \lambda, \mu>\lambda}{s-\lim _{\mu}} E_{\mu}$ for all $\lambda \geqq 0$,

c) $\mathbb{1}=\underset{\lambda \rightarrow+\infty}{s-\lim } E_{\lambda}$.

Let $T$ be the selfadjoint operator defined by this family:

$$
T=\int_{0}^{+\infty} \lambda d E_{\lambda} .
$$

For any $A$ and $B$ in $\mathfrak{N}_{\varphi}$ one has:

$$
\begin{aligned}
\left(T E_{\lambda} \Lambda_{\varphi} A, \Lambda_{\varphi} B\right) & =\int_{0}^{\lambda} \lambda^{\prime} d\left(E_{\lambda^{\prime}} \Lambda_{\varphi} A, \Lambda_{\varphi} B\right) \\
& =\int_{0}^{\lambda} \lambda^{\prime} d \varphi\left(B^{*} E_{\lambda^{\prime}} A\right) \\
& =\varphi\left(B^{*} f E_{\lambda} A\right) \\
& =\left(f E_{\lambda} \Lambda_{\varphi} A, \Lambda_{\varphi} B\right) .
\end{aligned}
$$

Hence $T E_{\lambda}=f E_{\lambda}$ for all $\lambda \geqq 0$.

For all $A$ in $\mathfrak{N}_{\varphi}$ :

$$
\begin{aligned}
\left\|T^{\frac{\varphi}{2}}\left(E_{\lambda}-E_{\mu}\right) \Lambda_{\varphi} A\right\|^{2} & =\left\|f^{\frac{1}{2}}\left(E_{\lambda}-E_{\mu}\right) \Lambda_{\varphi} A\right\|^{2} \\
& =\varphi\left(A^{*} f\left(E_{\lambda}-E_{\mu}\right) A\right) \\
& =\psi_{\mu}\left(A^{*}\left(E_{\lambda}-E_{\mu}\right) A\right)
\end{aligned}
$$

which tends to zero if $\lambda, \mu \rightarrow+\infty$.

Hence $\Lambda_{\varphi} A$ is in the domain of $T^{\frac{1}{2}}$ and for all $A, B$ in $\mathfrak{N}_{\varphi}$ :

$$
\left(T^{\frac{1}{2}} \Lambda_{\varphi} A, T^{\frac{1}{2}} \Lambda_{\varphi} B\right)=\psi_{\mu}\left(B^{*} A\right) .
$$

Therefore $T$ is the unique density operator $T_{\mu}$ with respect to $\varphi$ associated with the state $\psi_{\mu}$.

We now have:

$$
\begin{aligned}
S(\mu) & =-\int f \log f d v \\
& =-\lim _{\lambda \rightarrow \infty} \psi_{\mu}\left(\log f\left(E_{\lambda}-E_{1 / \lambda}\right)\right) \\
& =-\lim _{\lambda \rightarrow \infty}\left(\log f\left(E_{\lambda}-E_{1 / \lambda}\right) \xi_{\mu}, \xi_{\mu}\right) \\
& =-\lim _{\lambda \rightarrow \infty}\left(\log T_{\mu}\left(E_{\lambda}-E_{1 / \lambda}\right) \xi_{\mu}, \xi_{\mu}\right) \\
& =-\left\langle\log T_{\mu}\right\rangle_{T_{\mu}} .
\end{aligned}
$$

Let $M, v$ and $M^{\prime}, v^{\prime}$ be two localisable measure spaces. Suppose there is a *-isomorphism $\gamma$ between the $W^{*}$-algebras $\mathscr{L}^{\infty}(M, v)$ and $\mathscr{L}^{\infty}\left(M^{\prime}, v^{\prime}\right)$ 
which carries the weight $\varphi$ on $\mathscr{L}^{\infty}(M, v)$ defined by the measure $v$ into the weight $\varphi^{\prime}$ on $\mathscr{L}^{\infty}\left(M^{\prime}, v^{\prime}\right)$ defined by $v^{\prime}$. For any normal state $\psi^{\prime}$ on $\mathscr{L}^{\infty}\left(M^{\prime}, v^{\prime}\right)$ the entropy $S\left(\psi^{\prime}\right)$ with respect to $v^{\prime}$ equals the entropy $S\left(\psi^{\prime} \circ \gamma\right)$ of the state $\psi^{\prime} \circ \gamma$ on $\mathscr{L}^{\infty}(M, v)$ with respect to $v$. This means that the entropy $S$ is invariant under this kind of isomorphisms of measure spaces (called strong isomorphisms in [3]).

Consider now a Hilbert space $\mathscr{H}$. A faithful normal semifinite weight $\varphi$ on the space $\mathscr{B}(\mathscr{H})$ of all bounded linear operators on $\mathscr{H}$ is defined by: $\varphi\left(A^{*} A\right)=\operatorname{Tr} A^{*} A$ for all $A \in \mathscr{B}(\mathscr{H})$.

Proposition 3.2. Let $\varphi$ be the trace on $\mathscr{B}(\mathscr{H})$. Any normal state $\psi$ on $\mathscr{B}(\mathscr{H})$ is almost majorised by $\varphi$. Let $T_{\psi}$ be the density operator associated with $\psi$. The entropy $S(\psi)$ of the state $\psi$ is finite if and only if $\left\langle\log T_{\psi}\right\rangle_{T_{\psi}}$ exists and in that case:

$$
S(\psi)=-\left\langle\log T_{\psi}\right\rangle_{T_{\psi}} .
$$

Proof. Let $\varrho$ be the density matrix on $\mathscr{H}$ for which $\psi(A)=\operatorname{Tr} \varrho A$ for all $A \in \mathscr{B}(\mathscr{H})$. Because

$$
\left\|\Lambda_{\varphi} A \varrho^{\frac{1}{2}}\right\|^{2}=\operatorname{Tr} \varrho A^{*} A \leqq \operatorname{Tr} A^{*} A=\left\|\Lambda_{\varphi} A\right\|^{2},
$$

the mapping $\Lambda_{\varphi} A \rightarrow \Lambda_{\varphi} A \varrho^{\frac{1}{2}}$ defines a bounded operator $h$. This operator $h$ is positive, belongs to $\pi_{\varphi}(\mathscr{B}(\mathscr{H}))^{\prime}$ and satisfies for any $A$ and $B$ in $\mathfrak{N}_{\varphi}$ :

$$
\psi\left(B^{*} A\right)=\left(h \Lambda_{\varphi} A, h \Lambda_{\varphi} B\right) .
$$

Hence $h^{2}$ is the density operator $T_{\psi}$ associated with $\psi$ and $\Lambda_{\varphi} \varrho^{\frac{1}{2}}$ is the unique vector $\xi$ in $\mathscr{H}_{\varphi}$ for which $T_{\psi}=\pi^{\prime}(\xi)^{2}$.

Let $\varrho=\sum \lambda_{n} G_{n}$ be the spectral decomposition of $\varrho$. Define bounded operators $E_{n}$ by: $E_{n} \Lambda_{\varphi} A=\Lambda_{\varphi} A G_{n}$ for all $A \in \mathfrak{N}_{\varphi}$. The operators $E_{n}$ are mutually-orthogonal projections in $\pi_{\varphi}(\mathscr{B}(\mathscr{H}))^{\prime}$ with sum $\mathbb{1}$. One has $h=\sum \lambda_{n}^{\frac{1}{2}} E_{n}$.

Now:

$$
\begin{aligned}
\left\langle\log T_{\psi}\right\rangle_{T_{\psi}} & =\lim _{n \rightarrow \infty}\left(\left(\log T_{\psi} \sum_{\substack{p=1 \\
\lambda p \neq 0}}^{n} E_{p}\right) \Lambda_{\varphi} \varrho^{\frac{1}{2}}, \Lambda_{\varphi} \varrho^{\frac{1}{2}}\right) \\
& =\lim _{n \rightarrow \infty} \sum_{p=1}^{n}\left(\log \lambda_{p}\right)\left(E_{p} \Lambda_{\varphi} \varrho^{\frac{1}{2}}, \Lambda_{\varphi} \varrho^{\frac{1}{2}}\right) \\
& =\lim _{n \rightarrow \infty} \sum_{p=1}^{n}\left(\log \lambda_{p}\right)\left(\Lambda_{\varphi} \varrho^{\frac{1}{2}} G_{p}, \Lambda_{\varphi} \varrho^{\frac{1}{2}} G_{p}\right) \\
& =\lim _{n \rightarrow \infty} \sum_{p=1}^{n} \lambda_{p} \log \lambda_{p} \\
& =\operatorname{Tr} \varrho \log \varrho \\
& =-S(\psi) .
\end{aligned}
$$


Propositions 3.1 and 3.2 give the same expression for the entropy of a system of classical statistical mechanics described by a measure space and for the entropy of a quantum mechanical system described by a Hilbert space of wave functions. This justifies the following definition:

Definition 3.3. Let $\varphi$ be a faithful normal semi-finite weight on a von Neumann algebra $\mathscr{M}$. Let $\psi$ be a normal state on $\mathscr{M}$ almost majorised by $\varphi$. Let $T_{\psi}$ be the density operator associated with $\psi$. The entropy $S(\psi)$ of the state $\psi$ with respect to the weight $\varphi$ is given by:

$$
S(\psi)=-\left\langle\log T_{\psi}\right\rangle_{T_{\psi}}
$$

if $\left\langle\log T_{\psi}\right\rangle_{T_{\psi}}$ exists. In the other case $S(\psi)$ is not defined.

\section{References}

1. Dixmier,J.: Les algèbres d'opérateurs dans l'espace hilbertien, Paris: GauthierVillars 1956

2. Perdrizet,F.: Eléments positifs relatifs à une algèbre hilbertienne à gauche. Comp. Math. 23, 25 (1971)

3. Segal,I.E.: Equivalences of measure spaces. Amer. J. Math. 73, 275 (1951)

4. Sakai,S.: $C^{*}$-algebras and $W^{*}$-algebras. Berlin-Heidelberg-New York: Springer 1971

5. Combes, F.: Poids sur une $C^{*}$-algèbre. I. Math. pures et appl. 47, 57 (1968)

6. Haagerup, U.: Normal weights on $W^{*}$-algebras. (Preprint)

Communicated by H. Araki

J. Naudts

Universitaire Instelling Antwerpen

Physics Department

Universiteitsplein 1

B-2610 Wilrijk, Belgium 